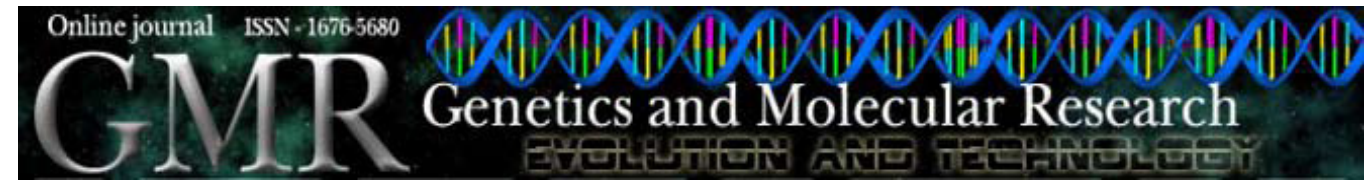

\title{
Loss of the AZFc region due to a human Y-chromosome microdeletion in infertile male patients
}

\author{
L.K. Pandey ${ }^{2}$, S. Pandey ${ }^{2}$, J. Gupta $^{1}$ and A.K. Saxena ${ }^{1}$ \\ ${ }^{1}$ Human Cytogenetic and Molecular Genetic Laboratory, Faculty of Medicine, \\ Centre of Experimental Medicine and Surgery, Institute of Medical Sciences, \\ Banaras Hindu University, Varanasi, India \\ ${ }^{3}$ Department of Obstetrics and Gynecology, Institute of Medical Sciences, \\ Banaras Hindu University, Varanasi, India \\ Corresponding author: A.K. Saxena \\ E-mail: draksaxena1@rediffmail.com
}

Genet. Mol. Res. 9 (2): 1267-1273 (2010)

Received March 5, 2010

Accepted April 9, 2010

Published June 29, 2010

DOI 10.4238/vol9-2gmr836

\begin{abstract}
Infertility is a major reproductive health threat; the frequency of male infertility due to Y-chromosome microdeletions is $13-18 \%$ in the human population; these microdeletions involve recurrent loss of three non-overlapping regions designated as AZFa, $\mathrm{AZFb}$ and AZFc, associated with spermatogenic failure. Several contradictory reports have been published regarding deletion frequency based on sequence-tagged site markers and genotypephenotype correlation. We examined the prevalence of Yq- deletion in 64 clinically diagnosed infertile male patients. We found a $3 \%$ frequency of microdeletion of the AZFc region; hormone profiles (FSH, LH and testosterone) showed significantly $(\mathrm{P}<0.001)$ elevated levels compared to controls. No mutations were observed in the AZFa
\end{abstract}


and $\mathrm{AZFb}$ regions, perhaps due to the selective use of sequencetagged site markers.

Key words: Male infertility; Y-chromosome microdeletion;

AZF regions; STS markers

\section{INTRODUCTION}

Genetic factors play a crucial role in about $10 \%$ of male infertility (Pryor et al., 1997). Human sperm are produced through a complex process of spermatogenesis involving the interaction of many genes and their expression (protein). Male infertility is classified into four major categories, namely azoospermia (complete absence of sperm), asthenozoospermia (low motility), oligozoospermia (low sperm count), and teratozoospermia (abnormal shape and size). In infertile couples, male factor alone contributes to approximately $50 \%$ of the cases including varicocele, obstruction of spermatic ducts, agglutination of sperms, impotency, and endocrine disorder.

Present knowledge has been amplified in the last two decades on male fertility genes assigned to the Y-chromosome, which contains approximately 60 billion bp, including testis determination factor. The quantitative role of genetic abnormalities in men is still unexplained due to the presence of several copies of genes assigned to Yq11.23 (Page et al., 1999). Several groups have reported that Yq deletions with DAZ-associated infertility are heterogeneous in some cases of spermatogenesis. The incidence of Y-chromosome microdeletion varies from 1 (van der Ven et al., 1997) to 50\% (Foresta et al., 1998) in idiopathic and non-idiopathic azoospermic or severe oligoazoospermic patients (Henegariu et al., 1994; Vogt et al., 1996). Recent studies on infertility-controlling spermatogenesis in men are involving azoospermic factors $(\mathrm{AZFc})$, present in the long arm of the Y-chromosome (Figure 1).

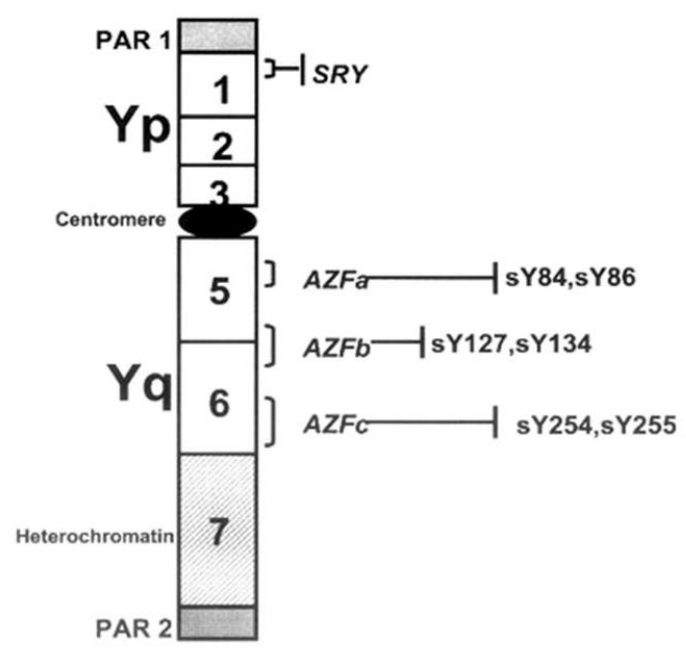

Figure 1. Schematic diagram of Y-chromosome showing AZF loci and their candidate gene associated with infertile male patients. 
Study of Yq microdeletion is quite relevant to the understanding of the mechanism of spermatogenesis, because the individuals are of different genetic background (Ma et al., 1993). The frequency of deleted regions is quite variable in different populations because of different environmental factors (Krausz et al., 2003). The AZFa and AZFb deletions are mostly associated with Sertoli-cell-only syndrome and arrest of spermatogenesis, respectively (Krausz et al., 2000). Partial or complete AZFc loss is associated with a highly variable heterogeneous group of phenotypes ranging from oligoazoospermic to Sertoli-cell-only syndrome (Calogero et al., 2001). The present study was designed to evaluate the prevalence of microdeletions of the Y-chromosome using GTG banding, hormonal profiles (LH, FSH and testosterone) and polymerase chain reaction (PCR)-based DNA analysis with sequencetagged sites (STS)-specific markers. Fertile men with normal sperm count were considered as the control group.

\section{MATERIAL AND METHODS}

Male patients were referred from the Department of Obstetrics and Gynecology to the Cytogenetic Laboratory, Centre of Experimental Medicine and Surgery, Institute of Medical Sciences, Banaras Hindu University, for molecular genetic analysis. The infertile patients $(\mathrm{N}=64)$ were diagnosed by spermiogram, hormone analysis and physical examination. The mean age range was 24-32 years and consisted of non-obstructive azoospermic, obstructive azoospermic and severely oligoazoospermic men (sperm count $<5$ million $/ \mathrm{mL}$ ). Semen analysis was performed in accordance with WHO (1992) guidelines, and controls were selected as normal healthy fertile males with a mean age range of 25-38 years.

Lymphocytes were cultured using a standard protocol of the laboratory for chromosomal analysis. Ethidium bromide $\left(10^{-4} \mathrm{M}\right)$ was added to the culture $2 \mathrm{~h}$ before harvesting for elongation of chromosomes. The cultures were finished by adding colchicine $(0.05 \mu \mathrm{g} / \mathrm{mL})$ $30 \mathrm{~min}$ before harvesting, and $0.075 \mathrm{M} \mathrm{KCl}$ was used as the hypotonic solution for $10 \mathrm{~min}$ at $37^{\circ} \mathrm{C}$. Cells were fixed in a methanol:acetic acid mixture for $15 \mathrm{~min}$ at room temperature. The slides were prepared by standard routine laboratory methods, and after banding, cells were stained with $5 \%$ Giemsa. At least 50 well-spread metaphases were used for karyotyping by a wide-field microscope for each data (Yunis et al., 1978).

Hormonal profiles were carried out as routine procedure using ELISA with specific antibodies. Serum FSH levels are more constant in peripheral blood, because FSH response to GnRH is delayed and of lesser magnitude, and clearance of FSH from blood is slow, compared with that of LH (Winters, 1994).

\section{PCR-based STS marker analysis}

Genomic DNA was extracted from peripheral blood $(0.5 \mathrm{~mL})$ using a DNA isolation kit (Bioneer, Korea) according to the manufacturer protocol. PCR was carried out in a 25$\mu \mathrm{L}$ reaction volume containing 50-100 ng template DNA, 1.5-2.0 mM dNTPs and 15 pmol of each primer, 10X standard buffers and $2 \mathrm{U}$ Taq DNA polymerase, used for amplification. PCR was performed on a My Genei 96 thermal cycler. Thermocycling consisted of an initial denaturation of $4 \mathrm{~min}$ at $96^{\circ} \mathrm{C}$, followed by 35 cycles of $1 \mathrm{~min}$ at $96^{\circ} \mathrm{C}, 30 \mathrm{~s}$ at $56^{\circ} \mathrm{C}$, and 1 min at $72^{\circ} \mathrm{C}$, with a final extension at $72^{\circ} \mathrm{C}$ for $5 \mathrm{~min}$. DNA from normal male individuals 
were included in each simplex (one set of primers/tube/individual) as controls. PCR products were separated on $2 \%$ agarose gels and visualized after staining with $1 \mu \mathrm{g} / \mathrm{mL}$ ethidium bromide. Gels were visualized by the Gel Doc system for characterization of bands. Screening of Y-chromosome microdeletions was carried out using specific STS markers with forward and reverse primers (Table 1). The primers were selected according to European Academy of Andrology guidelines. The set of primers allows the detection of more than $90 \%$ of deletions in three major AZF regions (Lucas et al., 2000).

\begin{tabular}{|c|c|c|c|}
\hline S. No & STS markers & Primers & Size (bp) \\
\hline 1 & sY156 & $\begin{array}{l}\text { (F) 5'-AGG AAC TGG CAG GAT TAG CC-3' } \\
\text { 5'- ATG TCA GGG TTT CCT TTG CC-3'(R) }\end{array}$ & 950 \\
\hline 2 & SPGY & $\begin{array}{l}\text { (F) 5'-TTT CAC ATA CAG CCA TTA AGT TTA GC-3' } \\
\text { 5'-ACA ATT TTG ATA GTC TGA ACA CAA GC-3'(R) }\end{array}$ & 460 \\
\hline 3 & sY254 & $\begin{array}{l}\text { (F) 5'-GGG TGT TAC CAG AAG GCA AA-3' } \\
\text { 5'-GAA CCG TAT CTA CCA AAG CAG C-3' (R) }\end{array}$ & 350 \\
\hline 4 & DAZLA3 & $\begin{array}{l}\text { (F) 5'-GAA TGC TGA ATT TTT ACT CTT GAA G-3' } \\
\text { 5'-CTC TAT ACG TGG CTA GAG TTC-3' (R) }\end{array}$ & 181 \\
\hline 5 & $92 \mathrm{R} 7$ & $\begin{array}{l}\text { (F) 5'-GAC CCG CTG TAG ACC TGA CT-3' } \\
\text { 5'-GCC TAT CTA CTT CAG TGA TTT CT-3'(R) }\end{array}$ & 709 \\
\hline
\end{tabular}

STS = sequence-tagged site

\section{Statistical analysis}

Statistical analysis was carried out by the chi-square test between controls and infertile patients to determine the level of significance.

\section{RESULTS}

Most of the infertile patients were sporadic cases with no apparent family history of fertility problems, while the remaining patients had a positive family history of infertility either in first- or second-degree relatives. Cytogenetic studies were carried out using GTG banding techniques and showed two karyotypes with 46,X,Yq- in azoospermic and severe oligoazoospermic patients.

Microdeletions of Y-chromosome AZFa-AZFc region were analyzed using PCRbased STS markers. Hence, interest has been generated to screen all three recurrently deleted non-overlapping subregions in proximal, middle, and distal Yq11, designated as AZFa, $\mathrm{AZFb}$ and AZFc, respectively. The present study showed that the prevalence of deletion of the AZFc region (sY254) was $3.33 \%$ in two patients with infertility but no deletion was apparently observed at the AZFa and AZFb loci. This study also did not show such mutation in oligoasthenozoospermic and teratozoospermic patients. Statistical analysis showed the lack of significant differences with respect to controls.

In serum hormone assay, there were significant differences in LH and FSH levels in obstructive azoospermic and severe oligozoospermic infertile males $(\mathrm{P}<0.001)$, as well as LH levels in teratoazoospermic patients $(\mathrm{P}<0.05)$, although no significant differences were observed in FSH and testosterone levels as shown in Table 2. 


Table 2. Hormonal profile study in different types of clinically diagnosed infertile male patients.
\begin{tabular}{llccc}
\hline S. No. & Types & & Hormonal level (means \pm SD) \\
\cline { 2 - 5 } & & LH & FSH & Testosterone \\
\hline 1 & Azoospermia & $16.49 \pm 9.84 * *$ & $37.19 \pm 33.62 * *$ & $3.82 \pm 2.02 * *$ \\
2 & Oligozoospermia (severe) & $5.41 \pm 5.10^{* *}$ & $7.14 \pm 6.83^{* *}$ & $4.20 \pm 1.62 * *$ \\
3 & Oligoasthenozoospermia & $12.76 \pm 9.87$ & $26.76 \pm 30.96$ & $3.73 \pm 1.67$ \\
4 & Teratozoospermia & $13.99 \pm 9.99 *$ & $30.32 \pm 32.30$ & $3.78 \pm 1.77$ \\
\hline$* * \mathrm{P}<0.001$ and $* \mathrm{P}<0.05$ & &
\end{tabular}

\section{DISCUSSION}

Several factors including lifestyle interactions between somatic and sex chromosome genes together contribute to the function of spermatogenesis (Lin et al., 1998) and the Yqchromosome deletions are the most common de novo cause of severe spermatogenetic defect (Vogt et al., 1995). The eukaryotic genome is highly fragile, and due to this instability, some crucial factors may alter the pathological state of infertility. It has been established that deletion of the long arm of the Y-chromosome is associated with spermatogenic failure (Tiepolo and Zuffardi, 1976). The immediate cause of reproductive failure in men with an extra marker is associated with spermatogenic disturbance leading to oligozoospermia. In the present study, the patients were classified on the basis of mean sperm count and morphology. This is associated with abnormal features of the sperm and decrease in number. The frequency of deletion varies from 1 to $55 \%$ in infertile men depending on inclusion criteria (Voget et al., 1996; Krausz et al., 2001), although the present study revealed 3.33\% aberrant karyotype with microdeletion of the AZFc region. Endocrine disorder is one of the parameters used as a diagnostic tool for the analysis of infertility (Sigman and Jarow, 1997). The elevated level of LH and FSH and low concentration of testosterone in our study may be one of the determinant factors for infertility, but a normal level of testosterone was found in a few cases of oligoazoospermic patients. Interestingly, the present study also showed that the loss of the AZFc region may be either because of common locus or natural transmission from father to child (Vogt et al., 1996; Pryor et al., 1997; Chang et al., 1999).

Environmental factors influence the variability in phenotypes of azoospermic and oligozoospermic patients. An advantage of this knowledge is to know how these gene families regulate meiotic events in fertility. It has been established that the deletion of AZFa leads to complete absence of germ cells and that $\mathrm{AZFb}$ is responsible for maturation arrest of meiosis while AZFc containing DAZ is specific for regulating spermatogenesis deletion that leads to azoospermia.

Tiepolo and Zuffardi (1976) first observed the microdeletion including the AZFc region (5.3\%) in azoospermic patients, although a similar locus was involved in the present infertile patients with little change in the frequency (3.33\%). Thangaraj et al. (2003) also observed the same frequency of AZFc (3.5\%) in azoospermic patients in the Indian population. The changes in the prevalence are due to either small sample size or endocrine disorders, or an unknown environmental factor may be important in influencing the frequency, as has also been documented in different populations of male infertility (Chiang et al., 2000; Kato et al., 2001; Madgar et al., 2002; Sawai et al., 2002; Tse et al., 2002). In the Caucasian population, the frequency of Y-chromosome microdeletion is small; 1\% (Pryor et al., 1997) and 3\% (Osterlund et al., 2000) have been reported in the Swedish population and 20\% in the New 
Zealand population (Kerr et al., 2000). The variation in the frequency of microdeletion in our study may be due to the use of selective STS markers (Martinez et al., 2000).

Cytogenetic and molecular studies of these cases suggest that no direct relationship exists between Y-microdeletions with hormone profile changes. In the Danish population, the FSH level seems to be quite high, although similar findings were obtained in the present study (Krausz et al., 2001; Frydelund-Larsen et al., 2002). The elevated level of FSH can be attributed to the failure of spermatogenesis in azoospermic patients in addition to Y-chromosome microdeletion. Endocrine assays further help to correlate our findings in men carrying deletion of the AZFc region. However, some cases of infertility cannot be explained by genetic alteration suggesting that infertility is a multifactorial disorders including environmental (nutritional) factor. This study revealed AZFc gene deletion in infertile cases with 46,X,Yq- karyotypes supporting the clinical observation of the occurrence of infertility in patients with normal testicular size, FSH level and sperm count.

Infertile patients who are carriers of Yq microdeletions and look for assisted reproductive techniques, such as in vitro fertilization and intracytoplasmic sperm injection (ICSI), will have the opportunity to become father. However, the significance and limitation of transmission of these abnormalities to male offspring should be fully explained (Page et al., 1999; Komori et al., 2002), because there is a possibility of loss of DNA repetitive sequences during ICSI techniques (Lupski, 1998). Some problems may occur with sex chromosome abnormalities, including an increased risk of miscarriage of affected embryos or in ICSI children. In conclusion, before adopting ICSI in infertile couples with severe spermatogenic problems, the significance of Yq microdeletion should be fully explained with regard to the treatment of infertile males in future generations.

\section{ACKNOWLEDGMENTS}

study.

Authors are thankful to the patients and their family who participated in the present

\section{REFERENCES}

Calogero AE, Garofalo MR, Barone N, De Palma A, et al. (2001). Spontaneous regression over time of the germinal epithelium in a Y chromosome-microdeleted patient: Case report. Hum. Reprod. 16: 1845-1848.

Chang PL, Sauer MV and Brown S (1999). Y chromosome microdeletion in a father and his four infertile sons. Hum. Reprod. 14: 2689-2694.

Chiang HS, Wei HJ and Chen YT (2000). Genetic screening for patients with azoospermia and severe oligo-asthenospermia. Int. J. Androl. 23: 20-25.

Foresta C, Ferlin A, Garolla A, Moro E, et al. (1998). High frequency of well-defined Y-chromosome deletions in idiopathic Sertoli cell-only syndrome. Hum. Reprod. 13: 302-307.

Frydelund-Larsen L, Krausz C, Leffers H, Andersson AM, et al. (2002). Inhibin B: a marker for the functional state of the seminiferous epithelium in patients with azoospermia factor C microdeletions. J. Clin. Endocrinol. Metab. 87: 5618-5624.

Henegariu O, Hirschmann P, Kilian K, Kirsch S, et al. (1994). Rapid screening of the Y chromosome in idiopathic sterile men, diagnostic for deletions in AZF, a genetic Y factor expressed during spermatogenesis. Andrologia 26: 97-106.

Kato H, Komori S, Nakata Y, Sakata K, et al. (2001). Screening for deletions in interval D16-22 of the Y chromosome in azoospermic and oligozoospermic Japanese men. J. Hum. Genet. 46: 110-114.

Kerr NJ, Zhang J, Sin FY, Benny P, et al. (2000). Frequency of microdeletions in the azoospermia factor region of the Y-chromosome of New Zealand men. N. Z. Med. J. 113: 468-470. 
Komori S, Kato H, Kobayashi S, Koyama K, et al. (2002). Transmission of Y chromosomal microdeletions from father to son through intracytoplasmic sperm injection. J. Hum. Genet. 47: 465-468.

Krausz C, Quintana-Murci L and McElreavey K (2000). Prognostic value of Y deletion analysis: what is the clinical prognostic value of $\mathrm{Y}$ chromosome microdeletion analysis? Hum. Reprod. 15: 1431-1434.

Krausz C, Rajpert-De ME, Frydelund-Larsen L, Quintana-Murci L, et al. (2001). Double-blind Y chromosome microdeletion analysis in men with known sperm parameters and reproductive hormone profiles: microdeletions are specific for spermatogenic failure. J. Clin. Endocrinol. Metab. 86: 2638-2642.

Krausz C, Forti G and McElreavey K (2003). The Y chromosome and male fertility and infertility. Int. J. Androl. 26: 70-75.

Lin WW, Lamb DJ, Lipshultz LI and Kim ED (1998). Absence of cyclic adenosine 3':5' monophosphate responsive element modulator expression at the spermatocyte arrest stage. Fertil. Steril. 69: 533-538.

Lucas H, Patrat C, Jouannet P, Beldjord C, et al. (2000). A novel, rapid, and accurate method for detecting microdeletion involving the DAZ gene in infertile men. Fertil. Steril. 73: 242-247.

Lupski JR (1998). Genomic disorders: structural features of the genome can lead to DNA rearrangements and human disease traits. Trends Genet. 14: 417-422.

Ma K, Inglis JD, Sharkey A, Bickmore WA, et al. (1993). A Y chromosome gene family with RNA-binding protein homology: candidates for the azoospermia factor AZF controlling human spermatogenesis. Cell 75: 1287-1295.

Madgar I, Green L, Kent-First M, Weissenberg R, et al. (2002). Genotyping of Israeli infertile men with idiopathic oligozoospermia. Clin. Genet. 62: 203-207.

Martinez MC, Bernabe MJ, Gomez E, Ballesteros A, et al. (2000). Screening for AZF deletion in a large series of severely impaired spermatogenesis patients. J. Androl. 21: 651-655.

Osterlund C, Segersteen E, Arver S and Pousette A (2000). Low number of Y-chromosome deletions in infertile azoospermic men at a Swedish andrology centre. Int. J. Androl. 23: 225-229.

Page DC, Silber S and Brown LG (1999). Men with infertility caused by AZFc deletion can produce sons by intracytoplasmic sperm injection, but are likely to transmit the deletion and infertility. Hum. Reprod. 14: 1722-1726.

Pryor JL, Kent-First M, Muallem A, Van Bergen AH, et al. (1997). Microdeletions in the Y chromosome of infertile men. N. Engl. J. Med. 336: 534-539.

Sawai H, Komori S and Koyama K (2002). Molecular analysis of the Y chromosome AZFc region in Japanese infertile males with spermatogenic defects. J. Reprod. Immunol. 53: 37-44.

Sigman M and Jarow JP (1997). Endocrine evaluation of infertile men. Urology 50: 659-664.

Thangaraj K, Gupta NJ, Pavani K, Reddy AG, et al. (2003). Y chromosome deletions in azoospermic men in India. $J$. Androl. 24: 588-597.

Tiepolo L and Zuffardi O (1976). Localization of factors controlling spermatogenesis in the nonfluorescent portion of the human Y chromosome long arm. Hum. Genet. 34: 119-124.

Tse JY, Yeung WS, Ng EH, Cheng LN, et al. (2002). A comparative study of Y chromosome microdeletions in infertile males from two Chinese populations. J. Assist. Reprod. Genet. 19: 376-383.

van der Ven, Montag M, Peschka B, Leygraaf J, et al. (1997). Combined cytogenetic and Y chromosome microdeletion screening in males undergoing intracytoplasmic sperm injection. Mol. Hum. Reprod. 3: 699-704.

Vogt PH, Edelmann A, Hirschmann P and Kohler MR (1995). The azoospermia factor (AZF) of the human Y chromosome in Yq11: function and analysis in spermatogenesis. Reprod. Fertil. Dev. 7: 685-693.

Vogt PH, Edelmann A, Kirsch S, Henegariu O, et al. (1996). Human Y chromosome azoospermia factors (AZF) mapped to different subregions in Yq11. Hum. Mol. Genet. 5: 933-943.

WHO (1992). WHO Laboratory Manual for the Examination of Human Semen and Semen-Cervical Mucus Interaction. 3rd edn. Cambridge University Press, Cambridge.

Winters SJ (1994). Endocrine evaluation of testicular function. Endocrinol. Metab. Clin. North Am. 23: 709-723.

Yunis JJ, Sawyer JR and Ball DW (1978). The characterization of high-resolution G-banded chromosomes of man. Chromosoma 67: 293-307. 